\title{
What and When: Parallel and Convergent Processing in Motor Control
}

\author{
Katsuyuki Sakai,, ${ }^{1,2}$ Okihide Hikosaka, ${ }^{1}$ Ryousuke Takino, ${ }^{3}$ Satoru Miyauchi, ${ }^{4}$ Matthew Nielsen, ${ }^{4}$ and \\ Tomoe Tamada 5 \\ ${ }^{1}$ Department of Physiology, Juntendo University School of Medicine, Tokyo 113, Japan, 2Department of Neurology, \\ Division of Neuroscience, Graduate School of Medicine, University of Tokyo, Tokyo 113, Japan, ${ }^{3}$ Shiraume Gakuen \\ College, Tokyo 187, Japan, ${ }^{4}$ Communications Research Laboratory, Kobe 651-24, Japan, and ${ }^{5}$ Exploratory Research for \\ Advanced Technology, Japan Science and Technology Corporation, Kyoto 619-02, Japan
}

Successful motor behavior requires making appropriate response (response selection) at the right time (timing adjustment). Earlier psychological studies have suggested that the response selection and timing adjustment processes are performed serially in separate stages. We tested this hypothesis using functional magnetic resonance imaging. The subjects performed a choice reaction time task in four conditions: two (on-line response selection required or not) by two (on-line timing adjustment required or not). We found that the neural correlates for the two processes were indeed separate: the anterior medial premotor cortex (presupplementary motor area) was selectively active in response selection, whereas the cerebellar posterior lobe was selectively active in timing adjustment. However, the functional separation was only partial in that the lateral premotor cortex and the intraparietal sulcus were active equally for response selection and timing adjustment. The lateral premotor cortex was most active when both processes were required, suggesting that it integrates the information on response selection and the information on timing adjustment; alternatively, it might contribute to the allocation of attentional resources during dual information processing. The intraparietal sulcus was equally active when either response selection or timing adjustment was required, suggesting that it modifies, rather than integrates, these processes. Furthermore, our results suggest that these activations related to response selection and timing adjustment were distinct from sensory or motor processes.

Key words: response selection; timing adjustment; motor execution; parallel processing; medial premotor cortex; cerebellum; lateral premotor cortex
Reaction process in response to an external stimulus has been thought to take several steps, starting from the stimulus encoding process to the final motor execution process (Fig. 1a; Frowein et al., 1981). This serial processing stage model is based on the additive factor theory (Sternberg, 1969), which assumes that the reaction time (RT) reflects a series of independent processing stages. Posner et al. (1973), Sanders (1977), and Frowein et al. (1981), using a choice reaction time task (CRT), have shown that uncertainty about which response to make (response uncertainty) and uncertainty about when to make the response (time uncertainty) both brought about an increase in the RT and that the effects were additive. The finding suggested that the two kinds of uncertainty affected two distinct processing stages, which have been referred to as the response selection and timing adjustment stages, respectively (Fig. $1 a$ ).

However, it is unknown whether the response selection and timing adjustment processes are performed in separate brain regions. Although a number of studies have suggested that some brain regions are related to response selection (Tanji and Kurata,

Received Sept. 2, 1999; revised Jan. 20, 2000; accepted Jan. 26, 2000.

This study was supported by Japan Society for Promotion of Science Research for the Future Program and Basic Research System Core. K.S. was supported by Japan Society for Promotion of Science Research Fellowship for Young Scientists. We are grateful to Hiroshi Imamizu and Mitsuo Kawato at Japan Science and Technology Corporation for their cooperation with SPM analysis.

Correspondence should be addressed to Katsuyuki Sakai, Department of Physiology, Juntendo University, School of Medicine, 2-1-1 Hongo, Bunkyo-ku, Tokyo 113, Japan. E-mail: katz@med.juntendo.ac.jp.

Copyright (C) 2000 Society for Neuroscience 0270-6474/00/202691-10\$15.00/0
1985; Kurata, 1993; Chen et al., 1995; Deiber et al., 1996; Humberstone et al., 1997) or timing adjustment processes (Ivry et al., 1988; Ivry and Keele, 1989; Perrett et al., 1993; Jueptner et al., 1995; Rao et al., 1997; Penhune et al., 1998), they were not intended to test the anatomical separation for the two processes. Crucial to prove the anatomical separation is to incorporate the two processes in a single experimental paradigm. In the present study, we have devised a CRT in which response uncertainty and time uncertainty were manipulated independently to change the amount of processing for response selection and timing adjustment. Functional magnetic resonance imaging (fMRI) was used to measure the brain activations while the normal human subjects performed the CRT. This factorially designed task procedure allowed us to test the anatomical separation and/or interaction of the response selection and timing adjustment processes. In addition, to identify the neural structures that are independent of the sensory modality of the stimuli, we tested auditory and visual paradigms and searched areas that were commonly active for the two paradigms.

\section{MATERIALS AND METHODS}

Subjects

Six normal human subjects (five men, one woman, ages 28-45 years, right-handed) participated in the study. Informed consents were obtained from all the subjects prior to the study. The experimental protocol was approved by the ethics committee of the Communications Research Laboratory. 
(a)

(b)
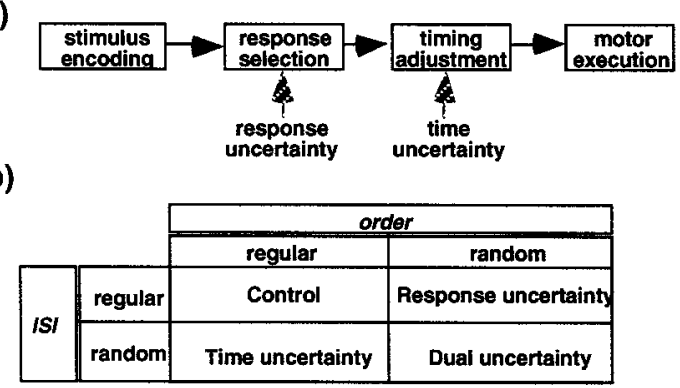

(c)

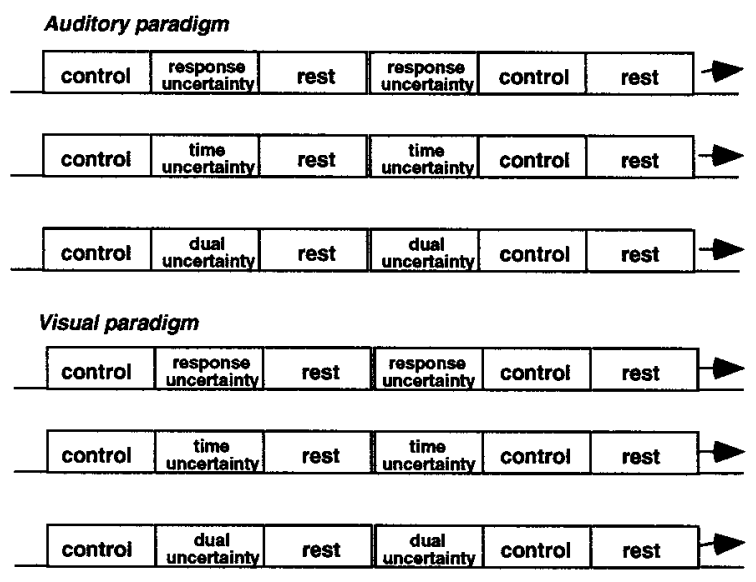

Figure 1. a, Scheme for reaction processes proposed in the earlier psychological studies (Frowein et al., 1981). The overall processes were thought to take several discrete stages arranged in a serial manner, where response uncertainty and time uncertainty independently affect the response selection and timing adjustment stages, respectively. In the original scheme, the timing adjustment was termed as motor adjustment. We used the present term to stress on the timing aspect. $b$, Task procedures. Subjects were asked to press buttons in response to the two types of stimuli using the index and middle finger of the right hand. The order of the presentation of the two types of stimuli and ISI were set either regular or random, creating four conditions named control, response uncertainty, time uncertainty, and dual uncertainty. $c$, Procedures for fMRI experiments. Six runs of experiments were conducted, three using the auditory paradigm, and the other three using the visual paradigm. For each run, one of the uncertainty, control, and rest conditions were repeated for six times in a counterbalanced order.

\section{Behavioral paradigm}

The subjects lay supine inside the bore of the magnet and held a plate equipped with two buttons. The subjects were asked to press one of the two buttons with the right index or middle finger in response to an auditory or visual stimulus while they were looking at a fixation spot at the center of the screen through a mirror. We conducted two sets of experiments, one using auditory stimuli, and the other using visual stimuli. For auditory stimuli, we used two types of tones with different pitches ( 1 and $2 \mathrm{kHz}$; rise-fall time, $5 \mathrm{msec}$; plateau, $20 \mathrm{msec}$; intensity, $95 \mathrm{~dB}$ ), which were presented through a headphone. For visual stimuli, we used two disk-shaped patterns with different colors (yellow or blue; diameter, $4^{\circ}$ in visual angle; duration of presentation, $60 \mathrm{msec}$ ), which were presented $2^{\circ}$ above the eye fixation spot on the screen.

The task was factorially designed such that the two factors, the order of the presentation of the two types of stimuli (order) and the interstimulus interval (ISI), were varied independently at two levels (regular/ random), creating four conditions named "control", "response uncertainty", "time uncertainty", and "dual uncertainty" (Fig. 1b). In control, the presentation of the two types of stimuli were alternated regularly and separated by a constant ISI of $1 \mathrm{sec}$. In response uncertainty, the two types of stimuli were presented in random orders but with a constant ISI of $1 \mathrm{sec}$. In time uncertainty, the two types of stimuli were presented in regular alternation with random ISIs ranging from 0.7 to $1.3 \mathrm{sec}$. In dual uncertainty, the two types of stimuli were presented in random orders with random ISIs $(0.7-1.3 \mathrm{sec})$. Two types of stimuli presented in regular alternation provide advance information about the next response, whereas stimuli presented at a constant interval provide advance information about the timing of button presses. In contrast to these predictable situations, the randomness in the order of stimulus presentation and/or ISIs creates unpredictable situations that require the increased amount of processing for response selection and/or timing adjustment. Thus, the comparison between the response uncertainty and control would reflect the response selection process, whereas the comparison between the time uncertainty and control would reflect the timing adjustment process. The comparison between dual uncertainty and control would reflect the combination of the two processes. This task design allowed us to determine how each brain area contributes to the response selection and the timing adjustment: nonselectively for both functions, selectively for one function, or selectively for the conjoint function.

During the task, the state of the ongoing task condition was indicated on the screen below the fixation spot, so that the subjects could make use of the advance information about the type of response and/or the timing of response. Subjects were instructed to suppress anticipatory responding and also to minimize choice errors and were trained for $20 \mathrm{~min}$ before the fMRI experiments. Trials with RTs $<100 \mathrm{msec}$ (anticipatory responding) and those with choice errors accounted for 2.2 and $3.4 \%$ of all the trials, which were excluded from the RT data analysis.

\section{Procedure of fMRI experiment}

First, structure images were obtained for each subject [fast low angle snap shot; repetition time (TR), $2.8 \mathrm{sec}$; echo time (TE), 4 msec; inversion time (TI), $300 \mathrm{msec}$; flip angle (FA), $15^{\circ}$; thickness, $1 \mathrm{~mm}$; resolution, $1 \times 1 \mathrm{~mm}$; matrix, $256 \times 256$ ). Subsequently, the subjects underwent six runs of fMRI experiments, three using auditory paradigm and the other three using visual paradigm (Fig. 1c). For each run, the subjects performed six blocks of the following three conditions: one of the three uncertainty conditions, control condition, and rest condition (Fig. 1c). In the rest condition, the subjects were asked only to focus their gaze on the fixation spot, and no stimuli were presented. Each condition block lasted for $35 \mathrm{sec}$. Within each run, the orders of the three conditions were counterbalanced across the six repetitions, and, also, the orders of the three runs for each of the auditory and visual paradigms were counterbalanced across the six subjects. For each run, the task condition was started after two dummy scans, whereas a time series of 126 scans (seven scans $\times$ three conditions $\times$ six repetitions) was performed at an interscan interval of $5 \mathrm{sec}$ using a $1.5 \mathrm{~T}$ scanner (Siemens Vision, Erlangen, Germany) equipped with a circular-polarized head coil. In each single scan, 14 slices of $\mathrm{T} 2 *$-weighted gradient-echo echoplanar images (TR, $5 \mathrm{sec}$; TE, $66 \mathrm{msec}$ FA, 90; thickness, $7 \mathrm{~mm}$; resolution, $1.8 \times 1.8 \mathrm{~mm}$; matrix, $128 \times 128)$ were collected parallel to the anterior and posterior commissure (AC-PC) line, which was determined based on the structure images obtained beforehand.

\section{Data analysis}

For data analysis, we used two methods. First, we performed statistical parametric analysis on the population data from the six subjects to identify the candidate brain regions related to response selection and/or timing adjustment processes. This analysis provides global estimates for the candidate brain regions and also shows the difference in the activation patterns between the two processes. However, the analysis depends on the population data and cannot take into account the anatomical variations across subjects. In addition, we needed another approach for statistical testing of the difference in the activations for each brain region between the two processes. Therefore, as the next step, we investigated whether the candidate regions are selectively related to response selection or timing adjustment process based on the individual data for each subject. In this second analysis, we delineated each candidate region based solely on the anatomical landmarks for each subject. This procedure could take the anatomical variations across subjects into account. Identification of neural correlates. Using SPM96 (http//:www.fil.ion.ucl. ac.uk/spm; Welcome Department of Cognitive Neurology, London, UK), the series of functional images for the six runs was realigned, normalized, and smoothed with a Gaussian filter of $4.5 \mathrm{~mm}$ full-width half-maximum. The time series of magnetic resonance signals for the two conditions of interest was cross-correlated with a boxcar reference function shifted for one data point $(5 \mathrm{sec})$, whereas the confounding effect of variation in global signal intensity across subjects was removed by analysis of covariance. Statistical parametric map of the $t$ statistics was 
constituted from the resulting voxel values and was transformed to the unit normal distribution (SPM $\{Z\}$; thresholded at $Z=3.09$ ). Taking spatial extent of activation into consideration, a corrected $p$ value of 0.05 was used as the final threshold for significance. To find the areas commonly active for the auditory and visual paradigms, we performed a conjunction analysis for the two paradigms (Price and Friston, 1997). The analysis first created a statistical parametric map that reflected the sum of the contrasts for the two paradigms and then eliminated regions where there were significant differences $(p<0.05)$ between the two. Thus, the procedure identifies areas that show significant difference in the activation between the two conditions of interest and that are independent of the sensory modality of the stimuli.

First, we identified the candidate brain regions related to response selection and/or timing adjustment processes. The conjunction analysis between the auditory and visual paradigms was performed respectively for the following three comparisons: response uncertainty versus control, time uncertainty versus control, and dual uncertainty versus control. Subsequently, the three statistical parametric maps were rendered onto the same standard brain template (Talairach and Tournoux, 1988) to show the areas active in any of the three comparisons. Thus, the map shows areas that were independent of the sensory modality of the stimuli and reflect (response uncertainty-control) $\cup$ (time uncertainty-control) $\cup$ (dual uncertainty-control). The identified areas would be related to response selection and/or timing adjustment processes.

Second, we identified the neural correlates for motor execution process. This process should be independent of the sensory modality of the stimuli, and, in addition, should be similarly involved in all of the three uncertainty conditions as well as control condition. Based on the idea, the conjunction analysis was initially performed between the auditory and visual paradigms, respectively for control versus rest, response uncertainty versus rest, time uncertainty versus rest, and dual uncertainty versus rest. Subsequently, we searched areas that were common to all of these four pairs of contrasts. Thus, the results should show areas that were independent of the sensory modality and reflect (control-rest) $\cap$ (response uncertainty-rest) $\cap$ (time uncertainty-rest) $\cap$ dual uncertainty-rest). The identified areas would be related to the motor execution process.

Testing the effect of uncertainty. For the next step, we tested the effect of the uncertainty about response and uncertainty about timing on the activations of the brain areas identified by the preceding analysis. As will be described in Results, we found the four candidate areas related to the response selection and/or timing adjustment processes [the presupplementary motor area/rostral cingulate motor area (PreSMA/rCMA), cerebellar posterior lobe (Cbll-post), lateral premotor cortex (PM), and an area around the intraparietal sulcus (IPS)] and the three candidate areas related to the motor execution process [the supplementary motor area proper (SMA-proper), primary motor cortex (M1), and cerebellar anterior lobe (Cbll-ant)].

The volumes of interest (VOIs) for these seven areas were determined for each subject based on the anatomical landmarks before the creation of activation maps according to the following criteria (see also Fig. $4 a$ ), and we calculated the sizes of active volumes within the VOIs as measures for the degree of activations.

PreSMA/rCMA: the rectangular portion over the medial premotor region, extending anteriorly for $25 \mathrm{~mm}$ from the plane passing the anterior commissure (VCA), and laterally for $10 \mathrm{~mm}$ from the midline to both sides (Picard and Strick, 1996). Additionally, the VOIs were separately determined for PreSMA and rCMA by assuming their border to be at the superior cingulate sulcus.

SMA-proper: the rectangular portion over the medial premotor region between the VCA and the coronal plane passing the posterior commissure (VCP) and above the superior cingulate sulcus (Picard and Strick, 1996).

Cbll-post: the portion of the cerebellum between the primary fissure and the horizontal fissure that corresponded to the H VI-VIIa (Larsell and Jansen, 1972).

Cbll-ant: the portion of the cerebellum anterior to the primary fissure.

PM: the area around the precentral sulcus above the junction of the precentral and inferior frontal sulci, extending anteriorly for $10 \mathrm{~mm}$ from the precentral sulcus and posteriorly to the midline between the precentral and central sulci (Fink et al., 1997).

M1: the area posterior to the midline between the precentral and central sulci, anterior to the central sulcus and above the junction of the precentral and inferior frontal sulci.

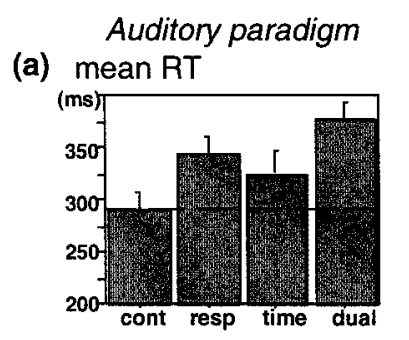

\section{Visual paradigm}

(b) variance of $\mathrm{RT}$
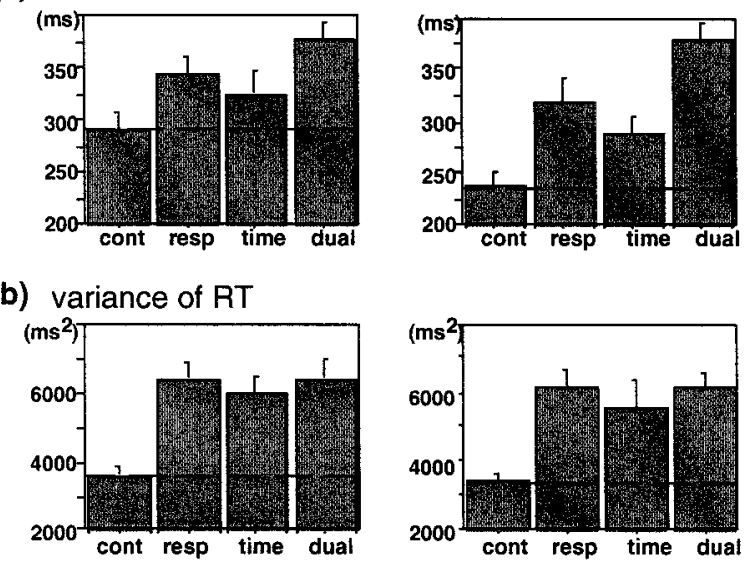

Figure 2. Reaction times $(a)$ and variance of reaction times $(b)$ for the four conditions: control (cont), response uncertainty (resp), time uncertainty (time), and dual uncertainty (dual). Means and the SEs for the six subjects were separately shown for the auditory (left) and visual (right) paradigms.

IPS: areas around the horizontal segment of the intraparietal sulcus, $10 \mathrm{~mm}$ medial or lateral to the sulcus.

Activation maps were created for each subject by comparing each of the control, response uncertainty, time uncertainty, and dual uncertainty conditions with the rest condition using the same statistical threshold as used in the population data analysis. Then, for each VOI, the active volume sizes were computed and expressed in cubic millimeters for the four conditions, on which two-factor ANOVA was performed to test the effect of uncertainty about response and uncertainty about timing.

Additionally, these measures of active volume sizes were used for statistical testing of the laterality of activations. For each area, the active volume sizes between the two hemispheres were compared using paired $t$ test, respectively for the four conditions.

Dependence on sensory modality. In addition to the areas commonly active for both auditory and visual paradigms, we identified areas that were dependent on the sensory modality of the stimuli. For this purpose, the statistical parametric maps were created respectively for the auditory and visual paradigms. The results were rendered onto the same standard brain template (Talairach and Tournoux, 1988) to see the difference and overlap of activations between the two paradigms. The comparisons were respectively made for response uncertainty versus control, time uncertainty versus control, and dual uncertainty versus control to identify the response selection and/or timing adjustment processes that were dependent on the sensory modality. In addition, the comparisons were respectively made for control versus rest, response uncertainty versus rest, time uncertainty versus rest, and dual uncertainty versus rest to identify the early perceptual processes that were unmodulated by any of the uncertainty conditions.

\section{RESULTS}

\section{Behavioral data}

Figure $2 a$ shows the RTs for button pressing under the four conditions: control, response uncertainty, time uncertainty, and dual uncertainty. For both auditory and visual paradigms, the RTs were longer in the three uncertainty conditions compared with the control. Two-factor ANOVA showed that both the response uncertainty and the time uncertainty increased the RT significantly (for auditory paradigm, the main effect of response uncertainty was $F_{(1,5)}=15.1, p<0.05$; the main effect of time uncertainty was $F_{(1,5)}=10.1, p<0.05$; for visual paradigm, they were $F_{(1,5)}=19.7, p<0.05$ and $F_{(1,5)}=6.68, p<0.05$, respectively). On the other hand, the interaction of the two kinds of uncertainty was not significant (auditory paradigm, $F_{(1,5)}=0.095$, $p>0.1$; visual paradigm, $\left.F_{(1,5)}=0.012, p>0.1\right)$. Relative to 
control, the increase of RT for dual uncertainty (mean increase of RT: 85.4 and $140.4 \mathrm{msec}$, respectively for auditory and visual paradigms) was close to the sum of the increase of RT for response uncertainty and $\mathrm{RT}$ for time uncertainty $(50.9+33.3$ and $70.8+50.3 \mathrm{msec}$, respectively, for auditory and visual paradigms).

To the contrary, for the variance of RT, the interaction of the two kinds of uncertainty was significant (auditory paradigm, $F_{(1,5)}$ $=24.1, p<0.05$; visual paradigm, $F_{(1,5)}=7.73, p<0.05$; Fig. $\left.2 b\right)$. Relative to control, the increase of the RT variance for dual uncertainty condition (mean increase of RT variance, 2813 and $2743 \mathrm{msec}^{2}$ ) was significantly shorter than the sum of the increase of RT variances for response uncertainty and time uncertainty $\left(2824+2380\right.$ and $\left.2717+2142 \mathrm{msec}^{2}\right)$. Thus, there was no additive effect on the variance of RT.

\section{fMRI data}

Using fMRI, we investigated whether different brain regions subserve the response selection and timing adjustment processes. We present the fMRI data in two parts: (1) show the candidate regions involved in response selection and/or timing adjustment; (2) examine whether the candidate regions are selectively related to response selection and/or timing adjustment. The results are shown separately for the areas that were independent of the sensory modality of the stimuli and for the areas that were dependent on the sensory modality of the stimuli.

\section{Identification of neural correlates}

Response selection and/or timing adjustment. We first determined the candidate brain regions related to response selection and/or timing adjustment. Initially, six statistical parametric maps for the group of six subjects were created by comparing each of the three uncertainty conditions with control, respectively for the auditory and visual paradigms. The coordinates of the active areas are indicated in Tables 1 and 2. To determine the areas that were independent of the sensory modality of the stimuli, the conjunction analysis was performed between the auditory and visual paradigms, respectively, for the three comparisons (coordinates shown in Table 3). The areas commonly active for the two paradigms were coded yellow on the surface of the standard brain (Fig. 3a, left) and on the axial sections (Fig. 3a, right). The figures indicate areas that were active in any of the three uncertainty conditions relative to control. The areas included the anterior part of the medial premotor cortex above and below the superior cingulate sulcus. They were located anterior to the VCA and thus were considered to be the PreSMA (Picard and Strick, 1996; Tanji, 1996) and the rCMA (Dum and Strick, 1993; Picard and Strick, 1996). Because the activation was continuous over the superior cingulate sulcus, the two areas were, hereafter, jointly referred to as PreSMA/rCMA. The yellow areas were also found in the lateral part of the cerebellar posterior lobe on both sides, which were mainly localized in H VI-VIIa (Larsell and Jansen, 1972) (Cbll-post) (better seen in Fig. 3a, right). Other areas coded in yellow were the PM (Passingham, 1993; Fink et al., 1997; Wise et al., 1997) and the area around the IPS. PreSMA/rCMA, Cbll-post, and PM were active on both sides, whereas IPS was active only on the left side. These areas were considered to be related to response selection and/or timing adjustment processes, irrespective of the sensory modality of the stimuli.

Motor execution. Note that the above activations were revealed with respect to the control condition. The comparison with the rest condition revealed a different set of brain areas, which were $\overline{\text { Table 1. Coordinates and } Z \text { scores of active areas in auditory paradigm }}$

\begin{tabular}{|c|c|c|c|}
\hline Comparison & Region & Coordinate & $Z$ scores \\
\hline \multicolumn{4}{|l|}{ Response uncertainty- } \\
\hline \multirow[t]{6}{*}{ Control } & PM & $-54,6,46$ & 4.88 \\
\hline & PreSMA/rCMA & $-6,6,52$ & 5.02 \\
\hline & & $10,12,50$ & 4.09 \\
\hline & IPS & $-40,-46,56$ & 4.25 \\
\hline & STG-post & $-70,-22,18$ & 4.44 \\
\hline & & $70,-26,24$ & 4.54 \\
\hline \multicolumn{4}{|l|}{ Time uncertainty- } \\
\hline \multirow[t]{6}{*}{ Control } & PM & $-50,4,42$ & 4.22 \\
\hline & IPS & $-40,-42,54$ & 4.25 \\
\hline & STG-post & $-68,-26,22$ & 4.92 \\
\hline & & $70,-30,20$ & 4.04 \\
\hline & Cbll-post & $-44,-76,-26$ & 5.16 \\
\hline & & $38,-62,-24$ & 4.11 \\
\hline \multicolumn{4}{|l|}{ Dual uncertainty- } \\
\hline \multirow[t]{10}{*}{ Control } & PM & $-52,6,48$ & 5.02 \\
\hline & & $-34,2,64$ & 5.11 \\
\hline & & $58,6,44$ & 4.68 \\
\hline & PreSMA/rCMA & $-4,4,54$ & 4.24 \\
\hline & & $4,6,50$ & 4.54 \\
\hline & IPS & $-34,-42,62$ & 4.44 \\
\hline & STG-post & $-56,-22,16$ & 4.23 \\
\hline & & $48,-24,18$ & 3.68 \\
\hline & Cbll-post & $-32,-56,-24$ & 3.83 \\
\hline & & $38,-62,-24$ & 4.11 \\
\hline$($ Control-Rest $) \cap$ & M1 & $-42,-18,48$ & 6.60 \\
\hline \multicolumn{4}{|c|}{ (Response uncertainty- } \\
\hline Rest $) \cap$ & SMA-proper & $-4,-6,60$ & 3.82 \\
\hline \multicolumn{4}{|l|}{ (Time uncertainty- } \\
\hline Rest) $\cap$ & IPS & $-42,-46,58$ & 4.25 \\
\hline \multicolumn{4}{|l|}{ (Dual uncertainty- } \\
\hline \multirow[t]{4}{*}{ Rest) } & STG-post & $-42,-22,18$ & 4.68 \\
\hline & A1 & $-50,-6,6$ & 5.12 \\
\hline & & $54,-10,8$ & 4.88 \\
\hline & Cbll-ant & $14,-46,-16$ & 6.72 \\
\hline
\end{tabular}

$\overline{\text { Cbll-ant, Cerebellar anterior lobe; Cbll-post, cerebellar posterior lobe; IPS, intrapa- }}$ rietal sulcus; M1, primary motor cortex; PM, lateral premotor cortex; PreSMA, presupplementary motor area; rCMA, rostral cingulate motor area; SMA-proper, supplementary motor area proper; STG-post, posterior superior temporal gyrus.

commonly active in the control and the three uncertainty conditions (Fig. $3 b$ ). The areas commonly active in the auditory and visual paradigms (shown in yellow) were the left medial premotor cortex posterior to VCA, which was considered to be the SMAproper (Picard and Strick, 1996; Tanji, 1996), the left M1, the right Cbll-ant, and the left IPS. These areas were considered to be related to the motor execution process because they were active independently of the sensory modality of the stimuli and were active irrespective of the presence or absence of the response/time uncertainty.

\section{Testing the effect of uncertainty}

The determination of active brain areas so far was still tentative because the analysis was based on the population data. To further characterize the candidate brain areas, we performed the second part of analysis: we delineated the candidate areas for each subject and statistically examined the contribution of these areas to the response selection and the timing adjustment.

After determining the VOIs on both hemispheres for each 


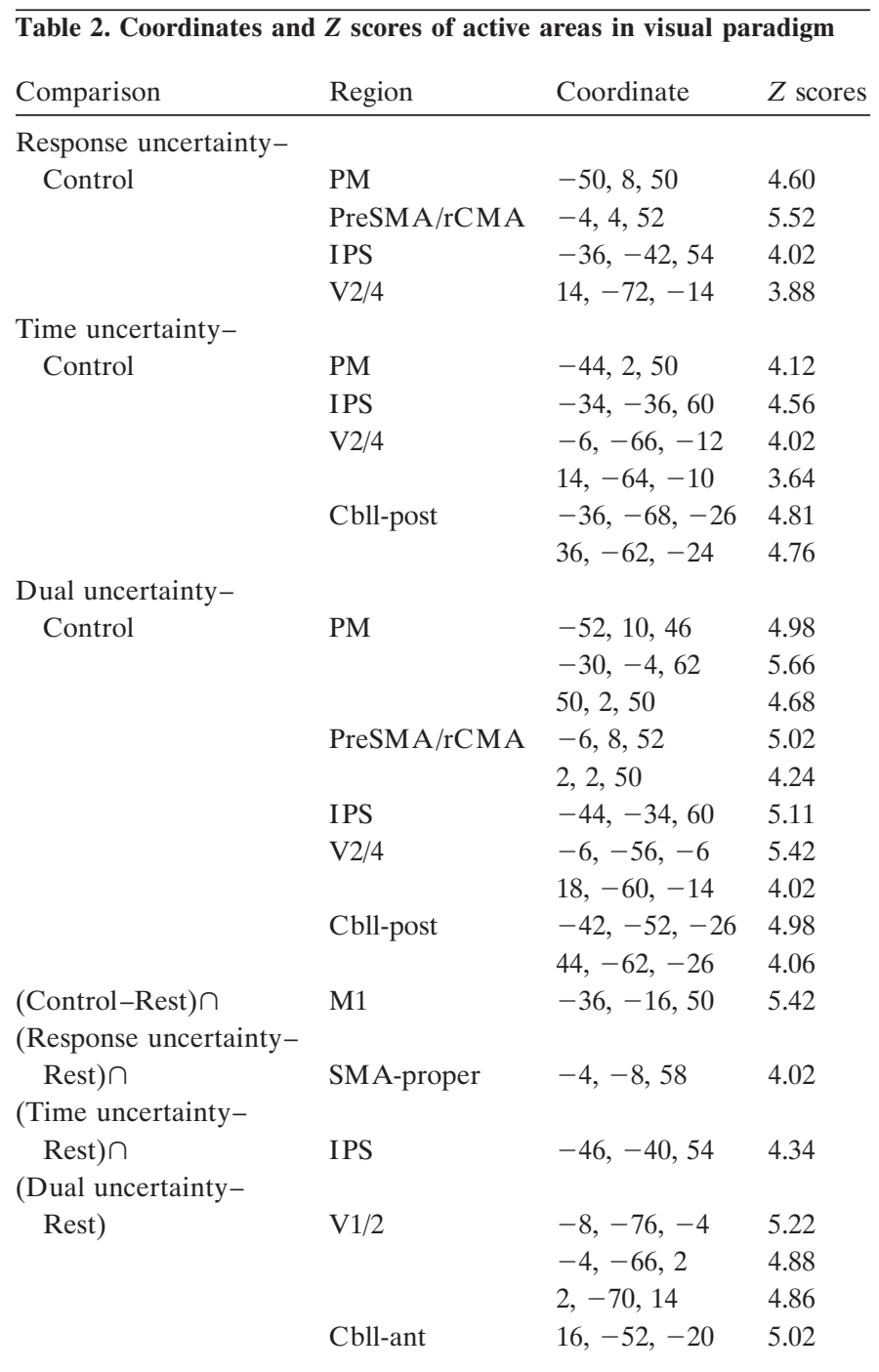

For abbreviations, see Table 1 legend.

subject (Fig. 4a), the active volume sizes within the VOIs relative to the rest condition were computed respectively for the four task conditions (control and the three uncertainty conditions) (Fig. 4b). Two-factor ANOVA performed on the active volume sizes showed that, for both the auditory and visual paradigms, the uncertainty about response produced significantly increased activation in PreSMA/rCMA, PM, and IPS $(p<0.05)$, whereas the uncertainty about timing produced significantly increased activation in Cbll-post, PM, and IPS ( $p<0.05)$ (Fig. 4b, Table 4). The interaction of the two factors was found to be significant only for PM, which showed highest activity under the dual uncertainty condition ( $p<0.05$; corrected for multiple comparisons) (Fig. $4 b$, Table 4). In contrast, the activations of SMA-proper, M1, and Cbll-ant were not affected either by the response or time uncertainty $(p>0.1)$.

The comparisons of the active volume sizes between the two hemispheres showed that, for all the conditions, PreSMA/rCMA and Cbll-post were bilaterally active (paired $t$ test; $p>0.1$ ), whereas PM and IPS was significantly more active on the left side $(p<0.01)$. Activations of the SMA-proper, M1, and Cbll-ant were unilateral: the former two confined on the left hemisphere, and the latter confined on the right side.
Table 3. Coordinates and $Z$ scores of commonly active areas for auditory and visual paradigms (Auditory $\cap$ Visual)

\begin{tabular}{llll} 
Comparison & Region & Coordinate & $Z$ scores \\
\hline Response uncertainty- & & & \\
Control & PM & $-52,2,50$ & 4.37 \\
& PreSMA & $-2,8,50$ & 4.51 \\
& & $6,12,54$ & 4.23 \\
IPS & $-36,-42,54$ & 4.03 \\
Time uncertainty- & & & \\
Control & PM & $-50,-4,48$ & 4.06 \\
& IPS & $-32,-44,56$ & 4.28 \\
& Cbll-post & $-32,-58,-22$ & 4.34 \\
& & $40,-62,-24$ & 4.51 \\
Dual uncertainty- & & & \\
Control & PM & $-50,2,52$ & 4.67 \\
& & $34,0,60$ & 4.56 \\
& PreSMA & $-2,10,52$ & 4.84 \\
& & $4,2,50$ & 4.09 \\
& IPS & $-36,-42,62$ & 4.52 \\
& Cbll-post & $-40,-56,-26$ & 4.82 \\
& & $40,-62,-24$ & 4.22 \\
Mest) & M1 & $-36,-16,46$ & 5.26 \\
(Control-Rest) $\cap$ & SMA-proper & $-4,-8,62$ & 4.32 \\
Response uncertainty- $\cap$ & & & \\
(Dime uncertainty- & IPS & $-46,-44,58$ & 3.88 \\
(Dual uncertainty- & & $16,-48,-18$ & 4.86 \\
\hline For & Cbll-ant & &
\end{tabular}

For abbreviations, see Table 1 legend.

\section{Dependence on sensory modality}

We also found brain activations that were selective for the sensory modality. Figure $5 a$ shows the areas that were active in each of the three uncertainty conditions, separately for the sensory modalities: selectively active for the auditory paradigm (shown in green), selectively active for the visual paradigm (shown in red), and commonly active in both paradigms (shown in yellow). The posterior portion of the superior temporal gyrus (STG-post; Rivier and Clarke, 1997) was selectively active for the auditory paradigm, whereas the ventral part of the occipital areas probably corresponding to V2 and V4 (Zeki et al., 1991; Sereno et al., 1995) was selectively active for the visual paradigm. As shown in Figure $5 a$, these sensory-modality-dependent areas were similarly active for the three uncertainty conditions.

Figure $5 a$ also confirmed the differential involvement of PreSMA/rCMA, Cbll-post, and PM in response selection, timing adjustment, and dual processes. PreSMA/rCMA was active for the response uncertainty, but not for the time uncertainty, whereas Cbll-post was active for the time uncertainty, but not for the response uncertainty. The two areas were active for the dual uncertainty. In contrast, PM was bilaterally active only for the dual uncertainty. Whereas PM was active on the left side for the response uncertainty and time uncertainty, its activation was significantly higher for the dual uncertainty compared to the single uncertainty, as seen from Figure $4 b$. The left IPS was active to a similar degree for the three uncertainty conditions.

Figure $5 b$ shows the areas that were active even without any uncertainty: the conjunction of the four comparison; (response uncertainty-rest), (time uncertainty-rest), (dual uncertaintyrest), (control-rest). Areas active for the auditory and visual 


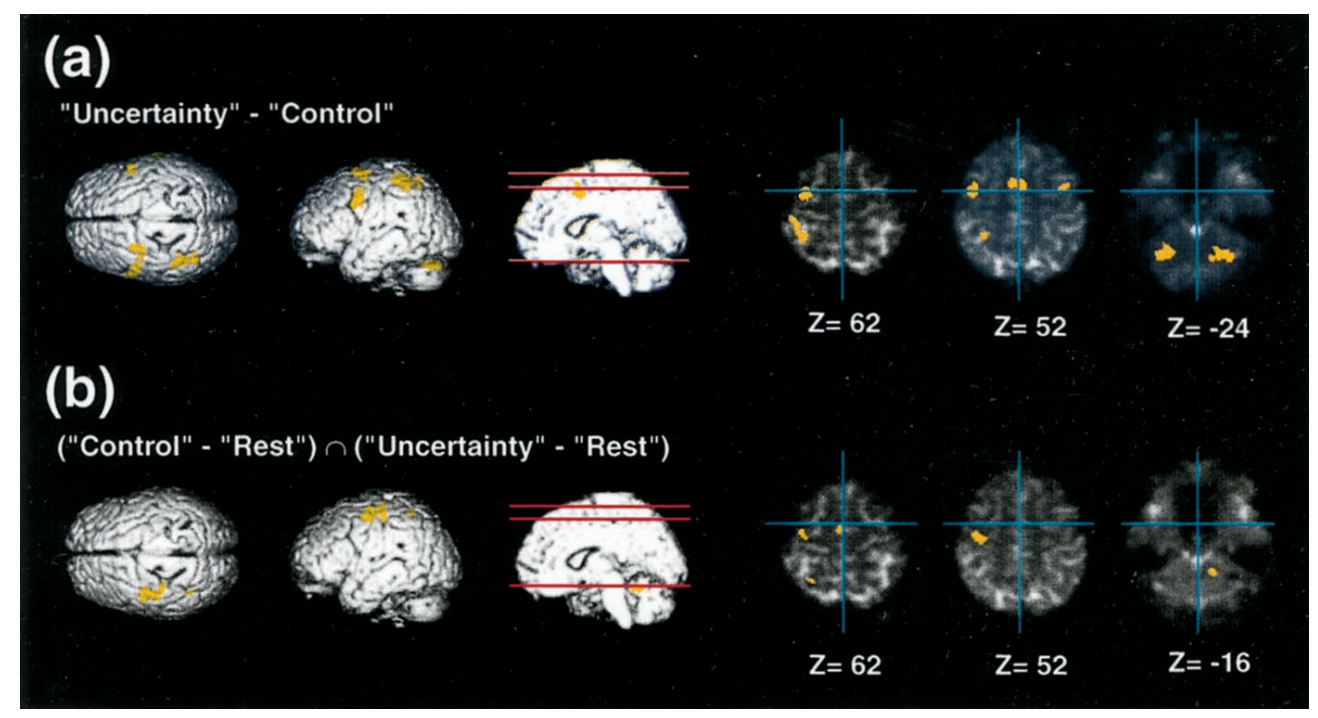

Figure 3. Statistical parametric analysis: sensory-modality-independent areas. Areas related to response selection and/or timing adjustment processes $(a)$ and areas related to motor execution process $(b)$ are shown in yellow on the surface of the standard brain (left three figures) and on the three axial slices of a single subject fitted into the Talairach space (Talairach and Tournoux, 1988) (right three figures). The left side of the figure indicates the left hemisphere. $a$, (response uncertainty-control) $\cup$ (time uncertainty-control) $\cup$ (dual uncertainty-control); $b$, (control-rest) $\cap$ (response uncertaintyrest) $\cap$ (time uncertainty-rest) $\cap$ (dual uncertainty-rest).

paradigms were respectively coded in green and red. As shown, the anterior portion of the superior temporal gyrus (A1) and STG-post were active only in the auditory paradigm (coded green), whereas the medial and ventral occipital areas including V1 and V2/V4 were active only in the visual paradigm (coded red).

\section{DISCUSSION}

By changing response uncertainty and time uncertainty independently, we found that a set of brain areas were active selectively or conjointly for the response selection and timing adjustment processes. The activations were largely distinct from those related to the motor execution processes that were present without any uncertainty and were also distinct from those related to the sensory processes that were selective for the sensory modality used for the response cue. Based on these results, we propose a parallel and convergent processing model as shown in Figure 6. In the following will be discussed the evidence for it and other possible interpretations of our findings.

\section{Separate processing}

The clear double dissociation between the PreSMA/rCMA and Cbll-post activations suggests independent neural computations for response selection and timing adjustment. This structural segregation makes it unlikely that the observed activations were merely the reflection of nonspecific attention. Instead, we consider that the activations reflect the on-line processing for selecting the appropriate response and that for determining the right timing of the response. Behaviorally, either process would lead to an increase in RT, as we found. However, it is unclear whether PreSMA/rCMA and Cbll-post actually compute the response and timing or they are related to the selective attention to stimulus features (Pardo et al., 1990) and timing.

PreSMA is anatomically and functionally distinct from the posteriorly situated SMA-proper (for review, see Picard and Strick, 1996; Tanji, 1996), and is particularly active when the subjects had to select the appropriate response on-line (Deiber et al., 1996; Humberstone et al., 1997; Petit et al., 1998; Ikeda et al., 1999; Sakai et al., 1999a), change or update motor plans (Matsuzaka and Tanji, 1996; Shima et al., 1996), or respond to an unpredictable visual stimulus (Dassonville et al., 1998). In our behavioral paradigms, the response was selected based on the auditory-motor or visuomotor mapping rule. This could be done by the direct connections from the auditory and visual association cortices (Luppino et al., 1993; Rizzolatti et al., 1998), as schematized in Figure 6. Alternatively, the information on the sensory cue may be relayed by way of other areas, such as the lateral prefrontal cortex (Bates and Goldman-Rakic, 1993; Luppino et al., 1993). rCMA behaved similarly to PreSMA (Table 4). This area has been shown to be connected with PreSMA (Luppino et al., 1993) and to play roles in higher-order motor control, especially in movement selection based on reward (Shima and Tanji, 1998).

Our data suggested that the posterior part of the cerebellum is related to the timing adjustment. Indeed, cerebellar patients show deficits in monitoring and reproducing timing (Ivry et al., 1988; Ivry and Keele, 1989; Nichelli et al., 1996). We now show, more specifically, that the timing-related area was localized bilaterally in the lateral part of the cerebellar posterior lobe, consistent with our preceding study (Sakai et al., 1998, 1999b). The finding is also consistent with the study of eyeblink conditioning showing that a part of Cbll-post (Larsell's H VI) plays a critical role in precise timing adjustment (Yeo and Hardiman, 1992; Gruart and Yeo, 1995). In our task the subjects might predict the time of the next cue stimulus and, if the actual time of the stimulus was different, adjust the timing of the response. Indeed, the RTs were shortest when the preceding ISI was around the mean value (1 sec) and increased when the preceding ISI was deviated from $1 \mathrm{sec}$ [mean RTs were 320, 271, and $310 \mathrm{msec}$, when the preceding ISI fell within the range of 700-900, 900-1100, and 1100-1300 msec, respectively (ANOVA, $F_{(5,10)}=14.36, p<0.01$ )]. This suggests that the activation of Cbll-post is related to the correction of timing error, consistent with the general view of the cerebellar function (Ito 1984; Thach et al., 1992; Kitazawa et al., 1998). Our data also suggest that both the auditory and visual information converge on the cerebellum (for review, see Schmahmann, 1996) (Fig. 6).

To summarize, PreSMA/rCMA and Cbll-post may contribute independently to the response selection and timing adjustment processes, or, in other words, to determine "what action to take" and "when to take it". However, these data alone cannot indicate whether the two processes were performed in a serial or parallel manner. The interaction of these processes must be examined to answer this question. 
(a)

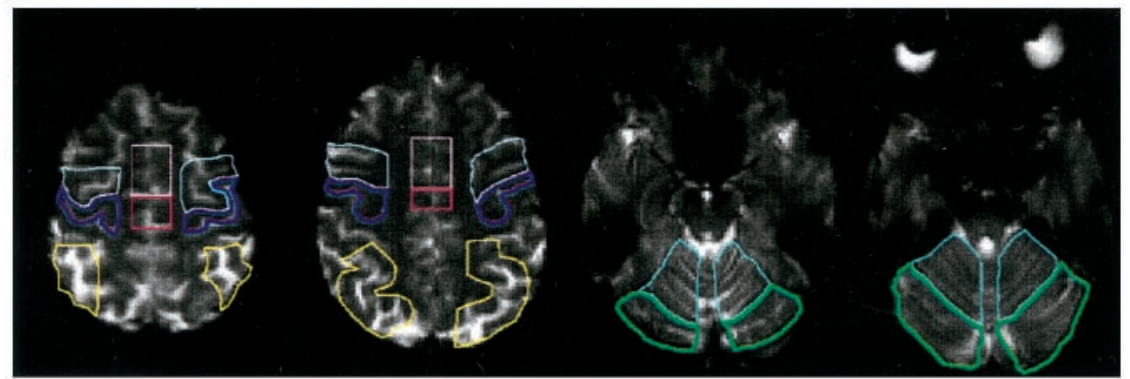

(b)

Auditory paradigm
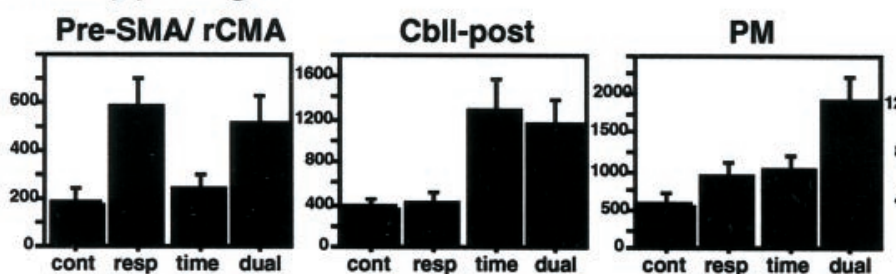

Cbll-ant
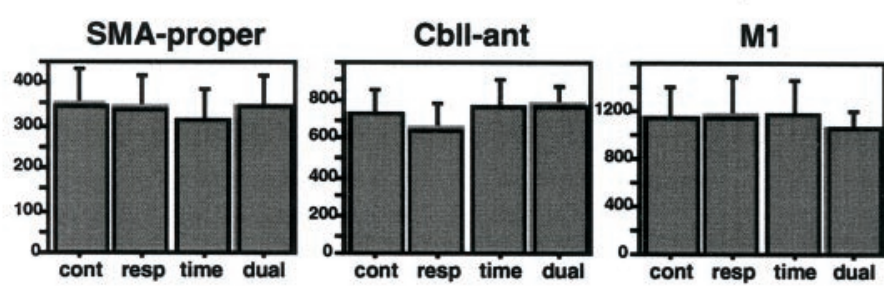

cont resp time dua

cont resp time dual

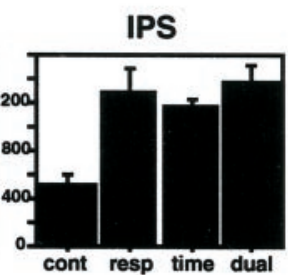

cont resp time dual

\section{Visual paradigm}
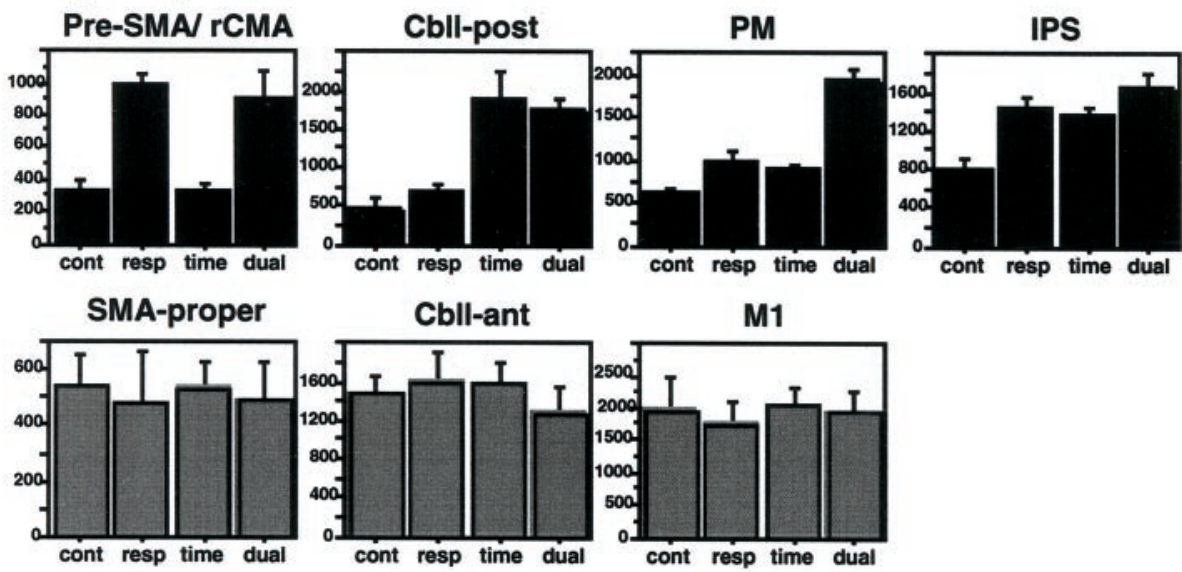

\section{Interaction}

PM was significantly more active in the dual uncertainty condition than in the single uncertainty conditions. The result suggests two possibilities. First, PM may play a role in integrating the information for response and timing. "What" information and "when" information may be processed in parallel by PreSMA/rCMA and Cbll-post, which, then, converge on PM to generate a final motor program (Fig. 6). Consistent with this view, PM receives connections from both the pre-SMA (Barbas and Pandya, 1987; Kurata, 1991) and the cerebellum (Middleton and Strick, 1997), although

it is unknown whether Cbll-post projects to PM. Also consistent is a physiological finding that neuronal activity in the dorsal PM was strongly influenced by the predictability of response and timing (Mauritz and Wise, 1986). As the second possibility, PM might contribute to the allocation of attentional resources during dual information processing (Iacoboni et al., 1998). In this case, PM would modify, rather than integrate, the response and timing processes (Fig. 6).

IPS was also related to both the response selection and timing adjustment processes. However, its activity was not enhanced in 
Table 4. Effect of response uncertainty and time uncertainty on active volume size

\begin{tabular}{llll} 
& Response & Time & Response $\times$ Time \\
\hline $\begin{array}{l}\text { Auditory paradigm } \\
\text { PreSMA/rCMA }\end{array}$ & $\underline{\underline{0.007}(19.3)}$ & $0.970(0.002)$ & $0.098(4.14)$ \\
Cbll-post & $0.832(0.050)$ & $\underline{0.012}(15.1)$ & $0.549(0.413)$ \\
PM & $\underline{0.011}(15.8)$ & $\underline{0.002}(34.5)$ & $\underline{0.038}(7.87)$ \\
IPS & $\underline{0.004}(25.4)$ & $\underline{0.038}(7.89)$ & $0.203(2.15)$ \\
PreSMA & $\underline{\underline{0.009}}(16.8)$ & $0.499(0.531)$ & $0.087(4.51)$ \\
rCMA & $\underline{\underline{0.022}}(10.7)$ & $0.584(0.342)$ & $0.356(1.04)$ \\
SMA-proper & $0.818(0.059)$ & $0.687(0.182)$ & $0.467(0.620)$ \\
M1 & $0.800(0.072)$ & $0.878(0.026)$ & $0.607(0.301)$ \\
Cbll-ant & $0.609(0.297)$ & $0.560(0.389)$ & $0.564(0.382)$ \\
Visual paradigm & & & \\
PreSMA/rCMA & $\underline{0.0001}(516)$ & $0.670(0.204)$ & $0.861(0.034)$ \\
Cbll-post & $0.892(0.020)$ & $\underline{0.002}(34.0)$ & $0.477(0.591)$ \\
PM & $\underline{0.002}(32.2)$ & $\underline{0.0001}(216)$ & $\underline{0.045}(7.07)$ \\
IPS & $\underline{0.011}(15.4)$ & $\underline{0.042}(7.41)$ & $0.116(3.62)$ \\
PreSMA & $\underline{0.0001}(106)$ & $0.252(1.68)$ & $0.319(1.23)$ \\
rCMA & $\underline{0.001}(51.2)$ & $0.377(0.939)$ & $0.600(0.314)$ \\
SMA-proper & $\underline{0.496}(0.539)$ & $0.967(0.002)$ & $0.954(0.004)$ \\
M1 & $0.435(0.721)$ & $0.762(0.102)$ & $0.728(0.136)$ \\
Cbll-ant & $0.693(0.175)$ & $0.679(0.192)$ & $0.375(0.950)$ \\
\hline
\end{tabular}

$P$ and $F$ values (within parentheses) for the effects of uncertainty about the response (Response), uncertainty about the timing (Time), and interaction of them (Response $\times$ Time) on the active volume sizes for the seven areas. For PreSMA and rCMA, the calculations were made on the combined values as well as the respective values. Underlines indicate the significant effect $(p<0.05)$.

For abbreviations, see Table 1 legend.

the dual uncertainty condition, suggesting that IPS does not play an integrative role. It has been shown that IPS is crucial for the selection of action (Snyder et al., 1997) and might encode the timing of events (Mackay and Crammond, 1987). Such selection signals and timing signals in IPS may be sent to the nonprimary sensory areas as top-down signals to improve their performance (Fig. 6). This can be regarded as an attentional mechanism in which IPS is considered to play an important role (Kalaska et al., 1983; Rushworth et al., 1997). Anatomically, IPS is densely connected with PM (Passingham, 1993; Wise et al., 1997; Rizzolatti et al., 1998), and may also be connected with PreSMA (Luppino et al., 1993) and the cerebellum (May and Andersen, 1986). IPS is also mutually connected with the nonprimary sensory areas (Blatt et al., 1990), consistent with our scheme (Fig. 6).

\section{Motor execution}

The present study has shown that the motor execution process is anatomically segregated from the response selection and timing adjustment processes, consistent with the suggestion in the earlier psychological study (Frowein and Sanders, 1978). The finding indicates the functional separation between PreSMA and SMAproper and between Cbll-post and Cbll-ant, which was consistent with the preceding studies (Ivry et al., 1988; Picard and Strick, 1996; Tanji, 1996; Allen et al., 1997; Sakai et al., 1998, 1999b). The only exception is IPS, which was active both in motor execution and in response selection/timing adjustment processes. It remains open to future studies whether different subareas within IPS are selectively involved in these processes.

\section{Perceptual processes}

Some of the nonprimary sensory areas were related to the response selection and the timing adjustment, but selectively for the
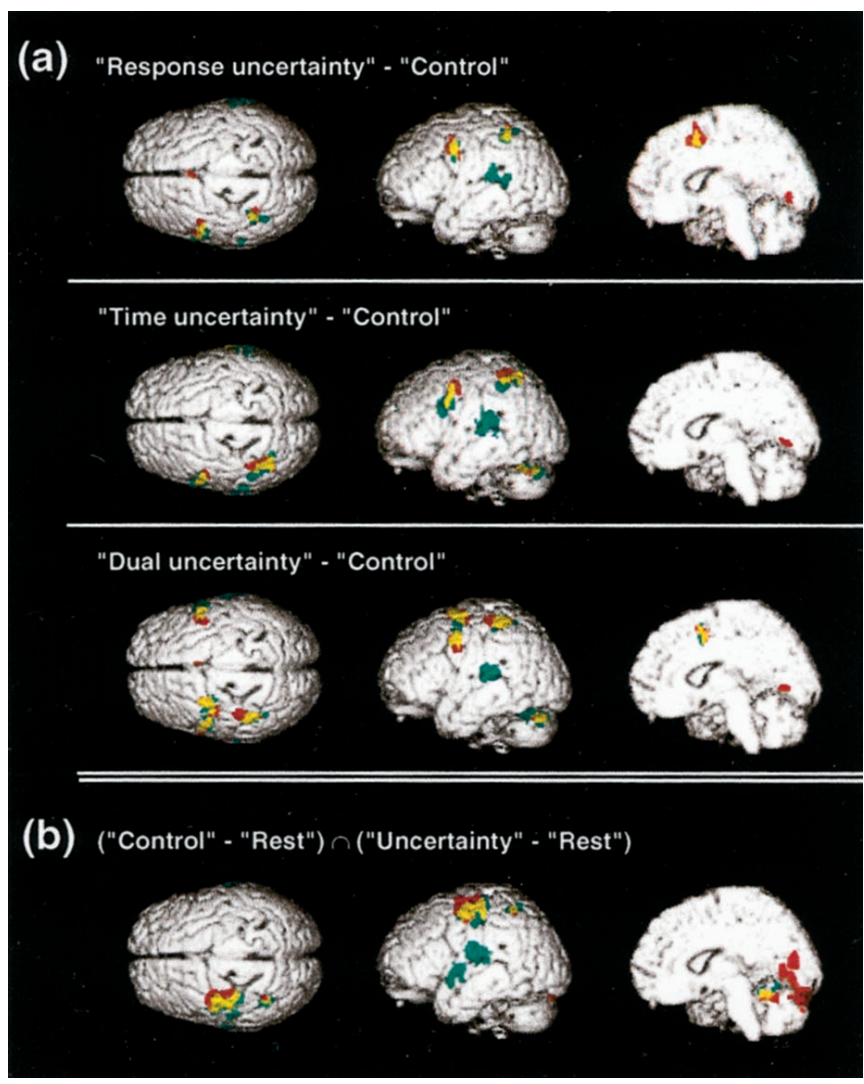

Figure 5. Statistical parametric analysis: sensory-modality-dependent areas. Areas of significant activation for the group of six subjects are indicated on the surface of the standard brain. $a$, Top row, (response uncertainty-control); second row, (time uncertainty-control); third row, (dual uncertainty-control); $b$, (control-rest) $\cap$ (response uncertaintyrest) $\cap$ (time uncertainty-rest) $\cap$ (dual uncertainty-rest).

sensory modality: STG-post for the auditory paradigm and V4 for the visual paradigm. The activation of these areas may reflect the increased level of attention to the auditory (especially, pitch) (Woodruff et al., 1996; Tzourio et al., 1997; Benedict et al., 1998) or visual (especially, color) stimuli (Moran and Desimone, 1985; McAdams and Maunsell, 1999). We speculate that the attentional effects were exerted by the IPS (Fig. 6). On the other hand, the primary sensory areas (A1 and V1) seemed to be unaffected by the uncertainty conditions (Woodruff et al., 1996; Zeki and Marini, 1998; McAdams and Maunsell, 1999), suggesting that these areas are related to the initial processing of stimulus encoding.

\section{Interpretation of the behavioral data}

The increase of RT for dual uncertainty was close to the sum of the increase of RT for response uncertainty and RT for time uncertainty. This additive effect seems to support the serial model (Fig. 1a). However, the result on the variance of RT is inconsistent with the serial model. The serial model would require a similar additive effect for the variance of RT: the variance of RT for dual uncertainty should be the sum of the RT variances for the two single uncertainty conditions (Sternberg, 1969). Our result indicated, on the contrary, that the variance of RT for dual uncertainty was close to the variance of RT for response uncertainty (Fig. 2b). According to the model shown in Figure 6, the response selection and timing adjustment processes are performed in parallel, but their integration starts only after both of 
Processing

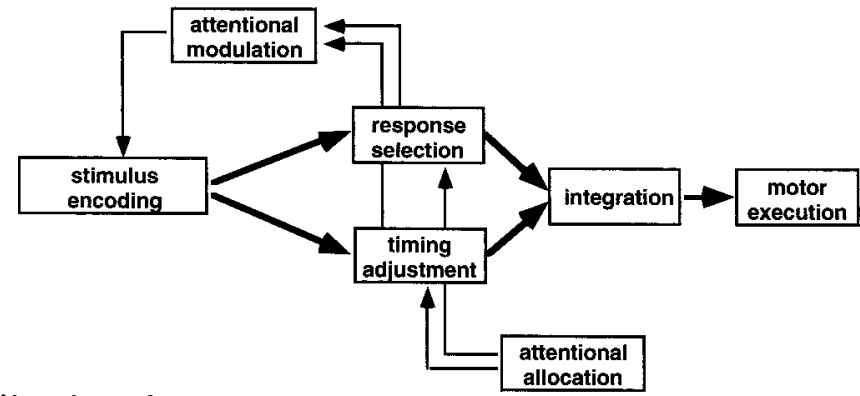

Neural correlates

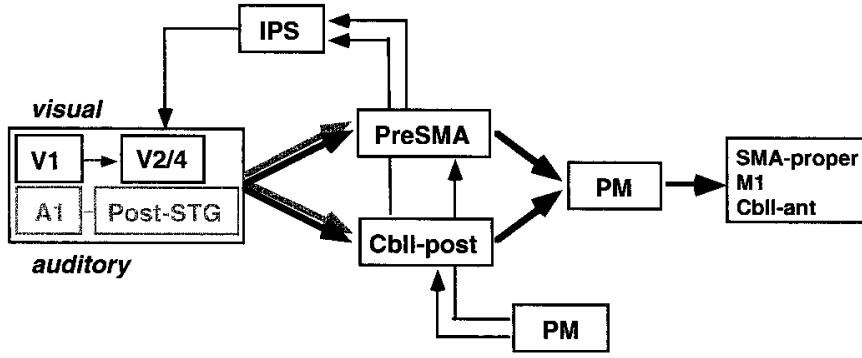

Figure 6. Hypothetical neural mechanisms for the processing of response selection and timing adjustment; the processing (top) and the neural correlates (bottom). The scheme depicts the parallel processing of response selection and timing adjustment, which are subserved by PreSMA/rCMA and Cbll-post. PM may either integrate these kinds of information or allocate attentional resources during dual information processing.

the two processes are finished. The variance of RT for dual processing would then be determined by the variance of RT for the slower process between the two (response selection in the present study). The additive effect of RT would be attributable to the time required for the integration of the two kinds of information.

\section{REFERENCES}

Allen G, Buxton RB, Wong EC, Courchesne E (1997) Attentional activation of the cerebellum independent of motor involvement. Science 275:1940-1943.

Barbas H, Pandya DN (1987) Architecture and frontal cortical connections of the premotor cortex (area 6) in the rhesus monkey. J Comp Neurol 256:211-228.

Bates JF, Goldman-Rakic PS (1993) Prefrontal connections of medial motor areas in the rhesus monkey. J Comp Neurol 336:211-228.

Benedict RHB, Lockwood AH, Shucard JL, Shucard DW, Wack D, Murphy BW (1998) Functional neuroimaging of attention in the auditory modality. NeuroReport 9:121-126.

Blatt GJ, Andersen RA, Stoner GR (1990) Visual receptive field organization and cortico-cortical connections of the lateral intraparietal area (area LIP) in the macaque. J Comp Neurol 299:421-445.

Chen Y-C, Thaler D, Nixon PD, Stern CE, Passingham RE (1995) The functions of the medial premotor cortex II. The timing and selection of learned movements. Exp Brain Res 102:461-473.

Dassonville P, Lewis SM, Zhu XH, Ugurbil K, Kim SG, Ashe J (1998) Effects of movement predictability on cortical motor activation. Neurosci Res 32:65-74.

Deiber M-P, Ibanez V, Sadato N, Hallett M (1996) Cerebral structures participating in motor preparation in humans: a positron emission tomography study. J Neurophysiol 75:233-247.

Dum RP, Strick PL (1993) Cingulate motor areas. In: Neurobiology of cingulate cortex and limbic thalamus (Vogt BA, Gabriel M, eds), pp 415-441. Boston: Birkhauser.

Fink GR, Frackowiak RSJ, Pietrzyk U, Passingham RE (1997) Multiple nonprimary motor areas in the human cortex. J Neurophysiol 77:2164-2174.

Frowein HW, Sanders AF (1978) Effects of stimulus degradation, S-R compatibility and foreperiod duration on choice reaction time and movement times. Bull Psychon Soc 12:106-108.

Frowein HW, Reitsma D, Aquarius C (1981) Effects of two counteracting stresses on the reaction process. In: Attention and performance IX (Long J, Baddely A, eds), pp 575-589. Hillsdale, NJ: Erlbaum.

Gruart A, Yeo CH (1995) Cerebellar cortex and eyeblink conditioning: bilateral regulation of conditioned responses. Exp Brain Res 104:431-448.

Humberstone M, Sawle GV, Clare S, Hykin J, Coxon R, Bowtell R, Macdonald IA, Morris PG (1997) Functional magnetic resonance imaging of single motor events reveals human presupplementary motor area. Ann Neurol 42:632-637.

Iacoboni M, Woods RP, Mazziotta JC (1998) Bimodal (auditory and visual) left frontoparietal circuitry for sensorimotor integration and sensorimotor learning. Brain 121:2135-2143.

Ikeda A, Yazawa S, Kunieda T, Ohara S, Terada K, Mikuni N, Nagamine T, Taki W, Kimura J, Shibasaki H (1999) Cognitive motor control in human pre-supplementary motor area studied by subdural recording of discrimination/selection-related potentials. Brain 122:915-931.

Ito M (1984) The cerebellum and neural control. New York: Raven.

Ivry RI, Keele SW (1989) Timing functions of the cerebellum. J Cogn Neurosci 1:134-150.

Ivry RI, Keele SW, Diener HC (1988) Dissociation of the lateral and medial cerebellum in movement timing and movement execution. Exp Brain Res 73:167-180.

Jueptner M, Rijntjes M, Weiller C, Faiss JH, Timmann D, Mueller SP, Diener HC (1995) Localization of a cerebellar timing process using PET. Neurology 45:1540-1545.

Kalaska JF, Caminiti R, Georgopoulos AP (1983) Cortical mechanisms related to the direction of two-dimensional arm movements: relations in parietal area 5 and comparison with motor cortex. Exp Brain Res 51:247-260.

Kitazawa S, Kimura T, Yin P-B (1998) Cerebellar complex spikes encode both destinations and errors in arm movements. Nature 392:494-497.

Kurata K (1991) Corticocortical inputs to the dorsal and ventral aspects of the premotor cortex of macaque monkeys. Neurosci Res 12:263-280.

Kurata K (1993) Premotor cortex of monkeys: set- and movementrelated activity reflecting amplitude and direction of wrist movements. J Neurophysiol 69:187-200.

Larsell O, Jansen J (1972) The comparative anatomy and histology of the cerebellum: the human cerebellum, cerebellar connections, and cerebellar cortex. Minneapolis: University of Minnesota.

Luppino G, Matelli M, Camarda R, Rizzolatti G (1993) Corticocortical connections of area F3 (SMA-proper) and area F6 (pre-SMA) in the macaque monkey. J Comp Neurol 338:114-140.

Mackay WA, Crammond DJ (1987) Neuronal correlates in posterior parietal lobe of the expectation of events. Behav Brain Res 24:167-179.

Matsuzaka Y, Tanji J (1996) Changing directions of forthcoming arm movements: neuronal activity in the presupplementary and supplementary motor area of monkey cerebral cortex. J Neurophysiol 76:2327-2342.

Mauritz K-H, Wise SP (1986) Premotor cortex of the monkey: neuronal activity in anticipation of predictive environmental events. Exp Brain Res 61:229-244.

May JG, Andersen RA (1986) Different patterns of corticopontine projections from separate cortical fields within the inferior parietal lobule and dorsal prelunate gyrus of the macaques. Exp Brain Res 63:265-278.

McAdams CJ, Maunsell JHR (1999) Effects of attention on orientationtuning functions of single neurons in macaque cortical area V4. J Neurosci 19:431-441.

Middleton FA, Strick PL (1997) Cerebellar output channels. Int Rev Neurobiol 41:61-82.

Moran J, Desimone R (1985) Selective attention gates visual processing in the extrastriate cortex. Science 229:782-784.

Nichelli P, Alway D, Grafman J (1996) Perceptual timing in cerebellar degeneration. Neuropsychologia 34:863-871.

Pardo JV, Pardo PJ, Janer KW, Raichle ME (1990) The anterior cingulate cortex mediates processing selection in the Stroop attentional conflict paradigm. Proc Natl Acad Sci USA 87:256-259.

Passingham RE (1993) The frontal lobes and voluntary action. Oxford: Oxford UP.

Penhune VB, Zattore RJ, Evans AC (1998) Cerebellar contributions to motor timing. J Cogn Neurosci 10:752-765.

Perrett SP, Ruiz BP, Mauk MD (1993) Cerebellar cortex lesions disrupt learning-dependent timing of conditioned eyelid responses. J Neurosci 13:1708-1718.

Petit L, Courtney SM, Ungerleider LG, Haxby JV (1998) Sustained 
activity in the medial wall during working memory delays. J Neurosci 18:9429-9437.

Picard N, Strick PL (1996) Motor areas of the medial wall: a review of their location and functional activation. Cereb Cortex 6:342-353.

Posner MI, Klein R, Summers J, Buggie S (1973) On the selection of signals. Mem Cognit 1:2-12.

Price CJ, Friston KJ (1997) Cognitive conjunctions: a new approach to brain activation experiments. NeuroImage 5:261-270.

Rao SM, Harrington DL, Haaland KY, Bobholz JA, Cox RW, Binder JR (1997) Distributed neural systems underlying the timing of movements. J Neurosci 17:5528-5535.

Rivier F, Clarke S (1997) Cytochrome oxidase, acetylcholinesterase, and NADPH-diaphorase staining in human supratemporal and insular cortex: evidence for multiple auditory areas. NeuroImage 6:288-304.

Rizzolatti G, Luppino G, Matelli M (1998) The organization of the cortical motor system: new concepts. Electroencephalogr Clin Neurophysiol 106:283-296.

Rushworth MFS, Nixon PD, Renowden S, Wade DT, Passingham RE (1997) The left parietal cortex and motor attention. Neuropsychologia 35:1261-1273.

Sakai K, Takino R, Hikosaka O, Miyauchi S, Sasaki Y, Pütz B, Fujimaki N (1998) Separate cerebellar areas for motor control. NeuroReport 9:2359-2363.

Sakai K, Hikosaka O, Miyauchi S, Sasaki Y, Fujimaki N, Pütz B (1999a) Pre-SMA activation during sequence learning reflects visuo-motor association. J Neurosci 19:RC1 (1-6).

Sakai K, Hikosaka O, Miyauchi S, Takino R, Tamada T, Iwata NK, Nielsen M (1999b) Neural representation of a rhythm depends on its interval ratio. J Neurosci 19:10074-10081.

Sanders AF (1977) Structural and functional aspects of the reaction process. In: Attention and performance VI (Dornic S, ed) pp 3-25. New York: Academic.

Schmahmann JD (1996) From movement to thought: anatomic substrates of the cerebellar contribution to cognitive processing. Hum Brain Mapp 4:174-198.

Sereno MI, Dale AM, Reppas JB, Kwong KK, Belliveau JW, Brady TJ, Rosen BR, Tootell RBH (1995) Borders of multiple visual areas in humans revealed by functional magnetic resonance imaging. Science 268:889-893.
Shima K, Tanji J (1998) Role for cingulate motor area cells in voluntary movement selection based on reward. Science 282:1335-1338.

Shima K, Mushiake H, Saito N, Tanji J (1996) Role for cells in the presupplementary motor area in updating motor plans. Proc Natl Acad Sci USA 93:8694-8698.

Snyder LH, Batista AP, Andersen RA (1997) Coding of intention in the posterior parietal cortex. Nature 386:167-170.

Sternberg S (1969) The discovery of processing stages: extensions of Donder's method. In: Attention and performance II (Koster WG, ed), pp 276-314. Amsterdam: North Holland.

Talairach J, Tournoux P (1988) Co-planar stereotaxic atlas of the human brain. New York: Thieme.

Tanji J (1996) New concepts of the supplementary motor area. Curr Opin Neurobiol 6:782-787.

Tanji J, Kurata K (1985) Contrasting neuronal activity in supplementary and precentral motor cortex of monkeys. I. Responses to instructions determining motor responses to forthcoming signals of different modalities. J Neurophysiol 53:129-141.

Thach WT, Goodkin HP, Keating JG (1992) The cerebellum and the adaptive coordination of movement. Annu Rev Neurosci 15:403-442.

Tzourio N, Massioui FE, Crivello F, Joliot M, Renault B, Mazoyer B (1997) Functional anatomy of human auditory attention studied with PET. NeuroImage 5:63-77.

Wise SP, Boussaoud D, Johnson PB, Caminiti R (1997) Premotor and parietal cortex: corticocortical connectivity and combinatorial computations. Annu Rev Neurosci 20:25-42.

Woodruff PWR, Benson RR, Bandettini PA, Kwong KK, Howard RJ, Talavage T, Belliveau RJ, Rosen BR (1996) Modulation of auditory and visual cortex by selective attention is modality-dependent. NeuroReport 7:1909-1913.

Yeo CH, Hardiman MJ (1992) Cerebellar cortex and eyeblink conditioning: a reexamination. Exp Brain Res 88:623-638.

Zeki S, Marini L (1998) Three cortical stages of colour processing in the human brain. Brain 121:1669-1685.

Zeki S, Watson JDG, Lueck CJ, Friston KJ, Kennard C, Frackowiak RSJ (1991) A direct demonstration of functional specialization in human visual cortex. J Neurosci 11:641-649. 